\title{
Vietnamese Immigrants in Brisbane, Australia: Perception of Parenting Roles, Child Development, Child Health, Illness, and Disability, and Health Service Utilisation
}

\author{
Uyen N. T. L. Tran \\ Department of Paediatrics \& Child Health, University of Qld, Queensland Health, C/O Inala Community Health Centre, P.O. Box 52, \\ Inala, QLD 4077, Australia \\ Correspondence should be addressed to Uyen N. T. L. Tran, uyen_tran@health.qld.gov.au \\ Received 13 June 2011; Accepted 2 December 2011 \\ Academic Editor: Cherylynn Bassani \\ Copyright (๑) 2012 Uyen N. T. L. Tran. This is an open access article distributed under the Creative Commons Attribution License, \\ which permits unrestricted use, distribution, and reproduction in any medium, provided the original work is properly cited. \\ The limited research into Vietnamese immigrants suggests that this group may have different perceptions relating to parenting \\ roles, child development, child health, illness, and disability, and differing patterns of health service utilisation. The author \\ conducted a pilot study exploring how Vietnamese immigrants differ from Anglo-Australian in relation to these issues. The pilot, \\ utilising a mixed quantitative and qualitative method, was conducted in Brisbane, Australia, with subjects being existing clients \\ of a health centre. Two focus group discussions were conducted and a structured questionnaire developed from the discussions. \\ Vietnamese immigrants in contrast to Australian-born Caucasians regard the general practitioner as the main health care provider \\ and were less satisfied with English-speaking health services. This study highlights potentially important health-related issues for \\ children of Vietnamese immigrants living in Brisbane, the importance of further research in this area, and the methodological \\ challenges faced when conducting research into Vietnamese immigrants.
}

\section{Introduction}

The literature indicates reduced access to health services and disparate health outcomes in people from culturally and linguistically diverse backgrounds (CLDBs) living in Western countries $[1,2]$. Low socioeconomic status, which is more prevalent in people from CLDB, accounts for some but not all of the disparate outcomes [3-5].

With Australia's increasingly cultural and linguistically diverse society, in particular a growing Asian population, health outcomes and health service utilisation of this population is an issue of great relevance to Australian practitioners and policy-makers. Even though commonalities may exist between CLDB populations, further exploration regarding poor health outcomes and access to health services demonstrate the importance of specific factors that are unique to individual communities.

During this study, staff at Inala Community Health's Department of Child, Youth and Family Health indicated possible deficits in the delivery of health services to families from CLDB, especially those from a Vietnamese background. Staff believed that these deficits may be related to different cultural perceptions held by parents about children's health, development, and the role of parenting. A greater understanding of cultural values and beliefs of people from CLDB, which underlie health behaviour, is essential if appropriate health services are to be provided.

A significant amount of the immigrant health research emanates from the United States of America, where health insurance status is often a major influencing factor in health access and service utilisation [6-9]. However given the differences between the American and Australian health care systems, American research is often not generalizable to an Australian context.

There have been few Australian studies which have explored health concerns and barriers to accessing health services amongst Vietnamese immigrants [10-12]. This literature indicates that families and women rate their children's health as a high priority and have concerns in relation to their children's general health, growth, and development and 
nutrition [11]. The Vietnamese family doctor is usually the first professional contact for any health-related problem [10]. The reasons cited for not utilising other mainstream health services include language barriers, inappropriate manner, and lack of cultural sensitivity of health professionals, transportation barriers, and waiting times $[10,11]$.

Traditional cultural health beliefs and practices are often incongruent with those of Western society and may pose a barrier to accessing health services [13]. Cultural differences in practices relating to pregnancy, birth, breast-feeding, and child birth [14-20] and children's oral health [13] have been identified in previous studies.

Thus this study and paper aims to contribute to the limited knowledge about Vietnamese immigrants living in Australia, their perceptions regarding their parenting roles, and the health and development of their children and their utilisation of health services for their children.

\section{Research Method}

2.1. Hypothesis. Prior to commencement of the study the following hypotheses were proposed.

(1) Vietnamese immigrant parents living in Brisbane have different perceptions about parenting roles and child health, illness, disability, discipline, behaviour, and child health services, compared with Australianborn Caucasian parents.

(2) Vietnamese immigrant parents living in Brisbane underutilise child health services, have less knowledge of them, are less able to access them, and are less satisfied with those they have used compared with Australian-born Caucasian parents.

2.2. Study Design. This is an exploratory study that utilised mixed methods of data collection, both quantitative (structured questionnaire) and qualitative (focus group) methodology. The focus groups were conducted first and responses were then used to inform issues to explore further in the questionnaire. For the purpose of this paper, the study group or participants will be referred to as the Vietnamese group or participants and the comparison group referred to as the Australian group or participants.

2.2.1. Setting. The study was conducted in Inala, Queensland in 2002. Inala is a suburb in the south-western outskirts of Brisbane. It is a low socioeconomic area with a culturally and linguistically diverse population and houses many recent immigrants [21]. The majority of Brisbane's Vietnamese community lives in Inala and its surrounding suburbs [21]. In 2001, persons of Vietnamese ancestry comprised the third commonest ancestry group in Inala (16.7\%).

2.2.2. Subjects. All subjects were existing clients of the Inala Community Health Centre. Inclusion criteria were adults over the age of 18 years, who lived in the Inala and surrounding area, gave informed consent, and had at least one child under the age of 5 years. The Vietnamese participants were first generation Vietnamese immigrants and the Australian group were Australian-born Caucasian. People from other cultural backgrounds were excluded to maintain cultural homogeneity. Exclusion criteria were known mental illness or intellectual disability in the adult subjects. The focus group and questionnaire participants were all existing clients of the Inala Community Health Centre. As this was a pilot and the intention is to follow-up with a full study, convenience sampling was used.

2.2.3. Focus Group. There were two focus groups, one with five Vietnamese immigrant parents and the other with nine Australian-born Caucasian parents. The chief investigator, who is bilingual in English and Vietnamese, and a child health nurse, facilitated both focus groups. A Vietnamese Liaison Officer was also present during the Vietnamese focus group.

2.2.4. Questionnaire. The questionnaire was developed by the Principal investigator using a well-accepted process described in the literature [22] and was based on issues which were uncovered from the focus group analysis. It comprised of standard question types including open-ended, dichotomous, forced-choice, rating questions using Likert scales [22] and vignettes [23]. The vignettes were created by the Principal investigator, who is a paediatrician. They were common scenarios in which the investigator has experienced cultural differences, between Vetnamese and Anglo-Australian parents, in clinical practice.

A pilot questionnaire was critically reviewed by a panel of paediatricians and professionals who work with the Vietnamese community to assess the questions for relevance. Professionals working with the Vietnamese community consisted of staff at the Inala Community health Centre as well as a Vietnamese general practitioner and a Vietnamese counsellor. The two native Vietnamese speakers, who are fluent in English, were asked to examine the questions for their ease of translation. Once the reliability of the questions had been confirmed, a qualified translator was asked to translate the questions into Vietnamese. They were then back translated into English for confirmation of meaning and equivalence [24].

The questionnaire was pretested by a small number of Vietnamese-born and Caucasian Australian-born parents to examine for content validity, ease of use, and relevance. The same panel of parents repeated the questionnaires two weeks later to examine retest reliability. The results were compared using correlation coefficients. The results from both the Australian and Vietnamese groups possessed high test-retest reliability, with a combined correlation coefficient of $84 \%$ (Pearson's) and 97.5\% (Spearman's). Second attempt score did not vary more than $8 \%$ different from first scores for each individual. The individual questions were also examined and were deemed to be answered consistently the second time compared with the first time.

The final questionnaire was administered to the Vietnamese and Australian study groups. The Vietnamese group were given the choice of the Vietnamese or English version, 
with all but one person choosing the Vietnamese version. There was one designated data collector for each group: a child health nurse for the Australian group and a Vietnamese Liaison Officer for the Vietnamese group. Although the questionnaires were self-administered, the designated data collectors were on hand to clarify any questions. The Vietnamese Liaison Officer, who is a qualified interpreter, assisted in interpreting the open-ended questions and vignettes in a culturally appropriate way and translating the data into English.

All information, consent forms, and the questionnaire were translated into Vietnamese by a qualified Vietnamese language translator and then back translated for accuracy. This is a methodologically sound and well-accepted approach in cross-cultural research [24].

\subsubsection{Recruitment}

Focus Groups. The Vietnamese focus group participants were selected from parents attending the Community Centre's Bacillus-Calmette-Guerin (BCG) Immunisation Clinic. They were approached by the Vietnamese liaison officer and gave verbal consent to participate in the focus group discussions. The Australian focus group participants were primarily parents who attended the Centre's parenting groups and baby clinics, who verbally agreed to participate in the focus group discussions.

Questionnaire. In recruiting participants for the questionnaire (See Supplementary Material available online at doi:10.1155/2012/932364), existing clients of Inala Community Health Centre's immunisation clinics, child health clinics, and parenting groups, with children between 0 and 5 years of age, were directly approached by the Centre's child health nurses and/or the Vietnamese Liaison Officer. The majority of the Vietnamese-born participants were clients attending the BCG immunisation clinics and the rest were clients of the Home Visiting Program. The participants were given an information sheet, either in English or Vietnamese, outlining the details and purpose of the study.

While there is some power gradient involved, as the parents are clients of the child health nurses and the interpreter, the nonthreatening nature of the study was explained. Using a Vietnamese recruiter helped to reduce the power gradient [25]. The participants were assured of confidentiality and anonymity and were informed of their right to refuse or participate. All parents signed a consent form.

2.3. Method of Analysis of Data. The focus group discussions were not taped because the literature indicates that this is not well accepted by Vietnamese women [10]. All efforts were made to accurately transcribe the main issues; however, without taping the sessions, content analysis was not possible. When analysing the responses for roles of health professionals, a panel of experts (two Caucasian Australianborn health care workers and two Vietnamese-born health care workers) independently scored the answers as correct, partly correct, or incorrect. The questions left unanswered were assumed to be incorrect (the participants who left the questions unanswered informed the Vietnamese interpreter that they did not know the answers). The vignette responses were also independently scored as correct, acceptable, or incorrect, by two paediatricians.

The questionnaire responses were entered into SPSS and descriptive statistics generated. The two study groups were examined in terms of demographic variables, utilisation of health services, satisfaction with health services, and understanding and perception of health professionals roles, common issues in child development and health, and causation of childhood conditions. For continuous variables one-way analysis of variance (ANOVA) and the Chi-squared tests for categoric variables were used.

Due to the small number of study participants, some of the variables were recoded with categories collapsed together. Frequency distribution curves were studied to determine which categories were to be collapsed together.

\section{Results}

Focus Groups. The main findings are summarised in Table 1. Discussions revolved around five main issues: health service utilisation, understanding of the roles of health professionals, child development issues, issues relating to parenting and discipline, and perception of causation of chronic disabilities and medical conditions.

Questionnaire. The questionnaire completion rate varied depending on the questions. The Vietnamese participants tended to leave questions blank if they did not know the answer or completed some of the questions about satisfaction with health services incorrectly, that is, attempted to answer question about a service they had not used.

3.1. Demographics. Table 2 compares the demographic characteristics of the two questionnaire study groups. The majority of participants were mothers and were in married or common-law relationships. The Australian group were slightly older, although this was not statistically significant. They had more children $(P=.024)$ who tended to be older $(P=.05)$, and these differences were statistically significant. In both groups most participants had not proceeded to tertiary education (only two Vietnamese participants had done some university studies and one Australian participant had completed university). The majority of participants were not in paid employment, with only one participant (Vietnamese) working outside the home.

More than half of the Vietnamese participants did not respond to the income question, but of those who responded, $60 \%$ had incomes below $\$ 20,000$ a year compared to only $11.1 \%$ of the Australian group, although this difference was not statistically significant.

$41.7 \%$ of the Vietnamese participants had lived in Australia for at least nine years, with the mean years in Australian 6.5 (SD 3.14), but most participants only rated 
TABLE 1: Focus group findings.

\begin{tabular}{|c|c|c|}
\hline Issue & Vietnamese & Australian \\
\hline \multirow{3}{*}{ Health Services } & Primarily GP ${ }^{1}$ & Use community health services and GP \\
\hline & Satisfied with GP & Less satisfied with GP \\
\hline & $\begin{array}{l}\text { Difficulty accessing community health services because } \\
\text { of transportation problems; want home visits }\end{array}$ & Want community centres to run fathers' groups \\
\hline \multirow{3}{*}{$\begin{array}{l}\text { Roles of health } \\
\text { professionals }\end{array}$} & Understood roles of GP, and paediatrician & Understood roles of GP, paediatrician, $\mathrm{CHN}^{2}, \mathrm{PT}^{3}$ \\
\hline & Confused roles of psychologist and psychiatrist & Confused roles of psychologist and psychiatrist \\
\hline & Did not know roles of $\mathrm{PT}, \mathrm{OT}^{4}, \mathrm{SLP}^{5}, \mathrm{CHN}$, and $\mathrm{SW}^{6}$ & Did not know roles of OT, SLP, and SW \\
\hline \multirow{3}{*}{ Child Development } & Offered child(ren) variety of toys & Emphasis on educational toys \\
\hline & Solid food introduced same time as Caucasian group & \\
\hline & $\begin{array}{l}\text { Allowed child to feed independently later for fear of } \\
\text { mess }\end{array}$ & \\
\hline Parenting & $\begin{array}{l}\text { Preferred explanation for discipline rather than } \\
\text { spanking }\end{array}$ & Mostly infants so discipline not as relevant \\
\hline
\end{tabular}

Causation of chronic

medical conditions, and

disabilities

$\begin{array}{lll}\text { Asthma } & \text { Cold, wind, allergies } & \text { Genetics, environment, grass, sand } \\ \text { Mental Illness } & \text { Trauma, use of } \mathrm{OCP}^{7} \text {, previous abortion, Karma } & \text { Genetics, stress } \\ \text { Epilepsy } & \text { Don't know } & \text { Genetics, trauma, drugs } \\ \text { ADHD }^{8} & \text { Don't know } & \text { Social factors, parenting, foods, preservatives } \\ \mathrm{CP}^{9} & \text { Don't know } & \text { Genetics, inutero problems, vitamin deficiencies }\end{array}$

${ }^{1}$ General Practitioner, ${ }^{2}$ Community Health Nurse, ${ }^{3}$ Physiotherapist, ${ }^{4}$ Occupational therapist, ${ }^{5}$ Speech and Language Pathologist, ${ }^{6}$ Social Worker, ${ }^{7}$ Oral contraceptive pill, ${ }^{8}$ Attention deficit hyperactivity syndrome, ${ }^{9}$ Cerebral Palsy.

their spoken English ability and understanding and ability to read English as "all right" or "very little or not at all".

Tables 3 and 4 compare the general health and community health services (out of all the child health services available at Inala Community Health Centre) utilised by the two groups, showing that the Vietnamese group used therapy services and information seminars significantly less than the Australian group. Of the community services, immunisation clinics, home visiting services, and antenatal services were the most utilised by Vietnamese participants.

Table 5 displays the results from the semantic differential questions, used to explore parental perception of various health services and professionals. Participants were asked to place an $\mathrm{X}$ on a visual analogue scale, corresponding to how their perceptions regard a particular service (see Supplementary Material). As the scale contained 5 boxes, the response was translated to a numerical value between " 1 " being most negative and " 5 " being most positive. A mean overall satisfaction score was calculated for each professional/service. Both groups held positive perceptions of general practitioners, hospitals, and community health centres.

The Vietnamese group were less positive about medical specialists, although this was not statistically significant. The Vietnamese group rated other health professionals (dentists, optometrists) less positively than the Australian group with the difference almost reaching statistical significance $(P=$ $.065)$. The two Vietnamese respondents rated therapists significantly less positively than the Australian group $(P=$ $.029)$. Both groups rated alternative practitioners quite positively on all qualities, but the number of respondents was small in both groups.

Table 6 displays the degree of satisfaction participants had with community child health services used, with "1" being the least satisfied and " 5 " being the most satisfied. However the number of respondents was very low for all services apart from home visiting, immunisation clinic, and antenatal clinic. The degree of satisfaction with all these services was high for both groups, with no statistical difference between the groups.

Table 7 tabulates participants' understanding of health professionals' roles, with the Vietnamese group having less knowledge than the Australian group of roles. In particular many in the Vietnamese group were unfamiliar with the roles of therapists. Also of note was the high rate confusion in both groups of psychiatrists and psychologists roles. There was a significant difference between the two groups in understanding the role of physiotherapist and speech pathologist, $P=.036$ and $P=.008$, respectively.

Table 8 outlines participant responses to the following vignettes: one, management of an acute diarrhoeal illness; two, approach to a child who is a picky eater; three, approach to an 18-month-old child who drinks only milk; four, approach to 2.5-year-old child who wants to feed independently; five, management of a 20-month-old child who is not walking; six, management of a 2-year-old child who is not talking; seven, perception regarding importance of tummy time when playtime; eight, perception regarding importance of books; and nine, perception regarding child playing in the mud. 
TABLE 2: Demographics of questionnaire participants.

\begin{tabular}{|c|c|c|c|}
\hline Variable & Vietnamese $n=12(\%)$ & Australian $n=10(\%)^{1}$ & $P$ \\
\hline \multicolumn{4}{|l|}{ Role } \\
\hline Mother & $12(100)$ & $10(100)$ & \multirow{3}{*}{ - } \\
\hline Father & $0(0)$ & $0(0)$ & \\
\hline$N$ Respondents (\%) & $12(100)$ & $10(100)$ & \\
\hline \multicolumn{4}{|l|}{ Age of parent (years) } \\
\hline Responders (\%) & $12(100)$ & $10(100)$ & \multirow{6}{*}{.16} \\
\hline$<30$ & $6(50)$ & $2(20)$ & \\
\hline$\geq 30$ & $6(50)$ & $8(80)$ & \\
\hline$N$ Respondents (\%) & $12(100)$ & $10(100)$ & \\
\hline Years in Australia (mean, SD) & $6.5(3.14)$ & Born here & \\
\hline$N$ Respondents (\%) & $12(100)$ & $10(100)$ & \\
\hline \multicolumn{4}{|l|}{ Marital status } \\
\hline Married/Common-Law & $8(80)$ & $9(90)$ & \multirow{3}{*}{.53} \\
\hline Other & $2(20)$ & $1(10)$ & \\
\hline$N$ Respondents (\%) & $10(83.3)$ & $10(100)$ & \\
\hline Number of children (mean, SD) & $1.83(0.72)$ & $2.70(0.95)$ & \multirow{2}{*}{.024} \\
\hline$N$ Respondents (\%) & $12(100)$ & $10(100)$ & \\
\hline Age children (mean, SD) & $2.13(2.13)$ & $3.75(1.33)$ & \multirow{2}{*}{.05} \\
\hline$N$ Respondents (\%) & $12(100)$ & $10(100)$ & \\
\hline \multicolumn{4}{|l|}{ Income $^{2}$} \\
\hline Low $(<\$ 20,000)$ & $3(60)$ & $1(11.1)$ & \multirow{4}{*}{.09} \\
\hline Middle (\$20,000-\$29,000) & $0(0)$ & $4(44.4)$ & \\
\hline $\operatorname{High}(>\$ 30,000)$ & $2(40)$ & $4(44.4)$ & \\
\hline$N$ Respondents (\%) & $5(41.6)$ & $9(90)$ & \\
\hline \multicolumn{4}{|l|}{ Level of education } \\
\hline Some secondary & $3(27.3)$ & $4(40)$ & \multirow{3}{*}{.66} \\
\hline Completed secondary & $8(72.7)$ & $6(60)$ & \\
\hline$N$ Respondents (\%) & $11(91.6)$ & $10(100)$ & \\
\hline \multicolumn{4}{|l|}{ Occupation } \\
\hline Home duties & $10(90.9)$ & $10(100)$ & \multirow{3}{*}{.52} \\
\hline Fulltime work & $1(9.1)$ & $0(0)$ & \\
\hline$N$ respondents $(\%)$ & $11(91.6)$ & $10(100)$ & \\
\hline
\end{tabular}

${ }^{1}$ One participant recruited was excluded from study analysis as it did not meet eligibility criteria.

${ }^{2}$ Based on Australian Bureau of Statistics Data Household Income and Income Distribution 2002-2003.

Both groups had a high rate of partially or fully correct responses for the vignettes, but the vignettes about delayed walking and delayed speech and language produced the greatest rate of incorrect responses from both groups. The Vietnamese group were statistically difference from the Australian group, with more incorrect responses for the vignettes about picky eater, feeding, and reading/books. The majority of participants in the Vietnamese group answered that they would "buy more books for their child but let her decide what she wanted to play with", for the vignette about reading and books. The majority of participants from both study group responded that they would "explain to their child that it was dinner time and it would be nice if she came", for the tantrum vignette, with only one participant (Australian) responding that they would take the child to "quite time" (time out).
Table 9 tabulates the responses of the two groups with regard to the causation of medical and neurodevelopmental conditions. Forced choice options were generated from the prior focus group discussions. Results have been presented descriptively, rather than as "correct" or "incorrect" answers, as some causes are debatable, and descriptive data give more insight into cultural beliefs around disease and illness. The Vietnamese group were more likely to answer that "upbringing" was a cause of Attention Deficit Hyperactivity Syndrome (ADHD) and were less likely to believe that "genetics" was a cause, with these differences being statistically significant. Additionally the Vietnamese group were less likely to respond that "genetics" was a cause of asthma, cerebral palsy (CP) or mental illness, with the former almost reaching statistical significance $(P=.056)$ and the latter being a statistically significant difference $(P=.004)$. Of note, one Vietnamese 
TABLE 3: Utilisation of health services by questionnaire participants.

\begin{tabular}{|c|c|c|c|}
\hline $\begin{array}{l}\text { Services } \\
\text { used-sometimes/often }\end{array}$ & $\begin{array}{c}\text { Vietnamese } \\
\quad N=12\end{array}$ & $\begin{array}{l}\text { Australian } \\
N=10\end{array}$ & $P$ \\
\hline \multicolumn{4}{|l|}{ General Practitioner } \\
\hline$N$ Utilising & $12(100)$ & $10(100)$ & \multirow{2}{*}{-} \\
\hline$N$ Respondents (\%) & $12(100)$ & $10(100)$ & \\
\hline \multicolumn{4}{|l|}{ Hospital } \\
\hline$N$ Utilising & $9(90)$ & $8(80)$ & \multirow{2}{*}{.50} \\
\hline$N$ respondents $(\%)$ & $10(83.3)$ & $10(100)$ & \\
\hline \multicolumn{4}{|c|}{ Community Health Centre } \\
\hline$N$ Utilising & $10(83.3)$ & $10(100)$ & \multirow{2}{*}{.50} \\
\hline$N$ respondents $(\%)$ & $10(100)$ & $10(100)$ & \\
\hline \multicolumn{4}{|l|}{ Medical Specialist } \\
\hline$N$ Utilising & $8(66.7)$ & $8(80)$ & \multirow{2}{*}{.42} \\
\hline$N$ respondents $(\%)$ & $12(100)$ & $10(100)$ & \\
\hline \multicolumn{4}{|l|}{ Health Professional } \\
\hline$N$ Utilising & $8(80)$ & $8(66.7)$ & \multirow{2}{*}{.42} \\
\hline$N$ respondents $(\%)$ & $12(100)$ & $10(100)$ & \\
\hline \multicolumn{4}{|l|}{ Therapist } \\
\hline$N$ Utilising & $1(9.1)$ & $7(70)$ & \multirow{2}{*}{.007} \\
\hline$N$ respondents (\%) & $11(91.7)$ & $10(100)$ & \\
\hline \multicolumn{4}{|l|}{ Alternative Practitioner } \\
\hline$N$ Utilising & $12(100)$ & $10(100)$ & \multirow{2}{*}{.41} \\
\hline$N$ respondents $(\%)$ & $2(16.7)$ & $3(30)$ & \\
\hline
\end{tabular}

TABLE 4: Use of services at inala community health centre.

\begin{tabular}{|c|c|c|c|}
\hline Services used & $\begin{array}{c}\text { Vietnamese } N(\%) \\
N \text { Respondents } \\
(\%)=12(100)\end{array}$ & $\begin{array}{c}\text { Caucasian } N(\%) \\
N \text { Respondents } \\
(\%)=10(100)\end{array}$ & $P$ \\
\hline Information seminars & 0 & $4(40)$ & .03 \\
\hline $\begin{array}{l}\text { Parent information } \\
\text { groups }\end{array}$ & 0 & $2(20)$ & .20 \\
\hline Home visits & $6(50)$ & $5(50)$ & .67 \\
\hline $\begin{array}{l}\text { Triple P (Parenting } \\
\text { workshops) }\end{array}$ & 0 & $3(30)$ & .08 \\
\hline Immunisation clinics & $9(75)$ & $5(50)$ & .22 \\
\hline Infant Feeding Clinic & $1(8.2)$ & $1(10)$ & .71 \\
\hline $\begin{array}{l}\text { Therapy (PT, OT, } \\
\text { SLP) }\end{array}$ & 0 & $5(50)$ & .01 \\
\hline Paediatrician clinic & $1(8.2)$ & $4(40)$ & .11 \\
\hline CYMHS $^{1}$ & $2(16.7)$ & $1(10)$ & .57 \\
\hline Antenatal clinic & $4(33.3)$ & $7(70)$ & .10 \\
\hline Day management & $1(8.3)$ & $2(20)$ & .43 \\
\hline Other & 0 & $1(10)$ & .46 \\
\hline
\end{tabular}

${ }^{1}$ Child, Youth, and Mental Health Services.

participant answered that "what one did in a previous life" was a cause of CP. The same participant also stated that this was a cause of epilepsy. Another Vietnamese participant stated that "abortion" (previous abortion) was a cause for mental illness in a woman.
TABLE 5: Satisfaction with health services used.

\begin{tabular}{|c|c|c|c|}
\hline \multirow{2}{*}{ Services used } & \multicolumn{2}{|c|}{ Mean (SD) [Respondents (\%)] } & \multirow{2}{*}{$P$} \\
\hline & Vietnamese & Australian & \\
\hline \multirow{2}{*}{ GP } & $4.45(0.69)$ & $4.47(0.49)$ & \multirow{2}{*}{.94} \\
\hline & {$[12(100)]$} & {$[10(100)]$} & \\
\hline \multirow{2}{*}{ Hospital } & $4.41(0.72)$ & $4.83(0.29)$ & \multirow{2}{*}{.14} \\
\hline & {$[12(100)]$} & {$[8(80)]$} & \\
\hline \multirow{2}{*}{ Com Health Cent } & $4.50(0.70)$ & $4.72(0.44)$ & \multirow{2}{*}{.42} \\
\hline & {$[12(100)]$} & {$[9(90)]$} & \\
\hline \multirow{2}{*}{ Medical Specialist } & $4.03(0.80)$ & $4.68(0.77)$ & \multirow{2}{*}{.14} \\
\hline & {$[6(50)]$} & {$[9(90)]$} & \\
\hline \multirow{2}{*}{ Health Professional } & $4.16(0.83)$ & $4.81(0.30)$ & \multirow{2}{*}{.065} \\
\hline & {$[5(41.6)]$} & {$[8(80)]$} & \\
\hline \multirow{2}{*}{ Therapist } & $4.00(0.00)$ & $4.84(0.42)$ & \multirow{2}{*}{.029} \\
\hline & {$[2(16.7)]$} & {$[7(70)]$} & \\
\hline \multirow{2}{*}{ Alternative Practitioner } & $4.33(0.58)$ & $4.67(0.58)$ & \multirow{2}{*}{.52} \\
\hline & {$[3(25)]$} & {$[3(30)]$} & \\
\hline
\end{tabular}

TABLE 6: Satisfaction with community child health services used.

\begin{tabular}{|c|c|c|c|}
\hline \multirow{2}{*}{ Services used } & \multicolumn{2}{|c|}{ Mean (SD) (Respondents (\%)) } & \multirow{2}{*}{$P$} \\
\hline & Vietnamese & Australian & \\
\hline \multirow{2}{*}{ Home Visiting } & $4.67(0.52)$ & $4.33(0.82)$ & \multirow{2}{*}{.27} \\
\hline & {$[6(50)]$} & {$[6(60)]$} & \\
\hline \multirow{2}{*}{ Immunisation Clinic } & $4.75(0.46)$ & $4.67(0.82)$ & \multirow{2}{*}{.41} \\
\hline & {$[8(66.67)]$} & {$[6(60)]$} & \\
\hline \multirow{2}{*}{ Feeding } & $5.00(0.00)$ & $5.00(0.00)$ & \multirow{2}{*}{ - } \\
\hline & {$[1(8.33)]$} & {$[3(30)]$} & \\
\hline \multirow{2}{*}{ Paediatrician } & $5.00(0.00)$ & $5.00(0.00)$ & \multirow{2}{*}{ - } \\
\hline & {$[1(8.33)]$} & {$[3(30)]$} & \\
\hline \multirow{2}{*}{ CYMHS } & $5.00(0.00)$ & $4.00(0.00)$ & \multirow{2}{*}{0.5} \\
\hline & {$[1(8.33)]$} & {$[1(10)]$} & \\
\hline \multirow{2}{*}{ Antenatal } & $4.75(0.500)$ & $4.86(0.38)$ & \multirow{2}{*}{.45} \\
\hline & {$[4(30)]$} & {$[7(70)]$} & \\
\hline
\end{tabular}

\section{Discussion}

This study has elicited important issues relating to Vietnamese immigrant and refugee parents' perception of child health, development, and their utilisation of child health services, with the intention of further exploration of these issues with a subsequent full-scale study.

The main study findings and implications are summarised in Tables 10 and 11. The findings do partly support the initial hypotheses that Vietnamese immigrant parents living in Brisbane have different perceptions about parenting roles and child health, illness, disability, discipline, behaviour, and child health services, compared with Australian-born Caucasian parents, and that they underutilise child health services, have less knowledge of them, are less able to access them, and are less satisfied with those they have used compared with Australian-born Caucasian parents. Two important themes emerged from this 
TABLE 7: Understanding of professionals' roles.

\begin{tabular}{lccc}
\hline Professional & \multicolumn{2}{c}{ Partially/fully correct } & $P$ \\
\hline Physiotherapist & $2(33.3)$ & $9(90)$ & .036 \\
Vietnamese $N$ Respondents (\%) & $6(50)$ & $11(100)$ & \\
Speech Pathologist & $2(33.3)$ & $10(100)$ & .008 \\
N Respondents (\%) & $6(50)$ & $10(100)$ & \\
Occupational & $2(33.3)$ & $7(70)$ & .18 \\
Therapist & $6(50)$ & $10(100)$ & \\
$N$ Respondents (\%) & $2(33.3)$ & $5(50)$ & .45 \\
Psychologist & $6(50)$ & $10(100)$ & \\
$N$ Respondents (\%) & $3(42.9)$ & $5(50)$ & .58 \\
Psychiatrist & $7(58.3)$ & $10(100)$ & \\
$N$ Respondents (\%) & $5(71.4)$ & $7(70)$ & .69 \\
Community Health & $7(58.3)$ & $10(100)$ & \\
Nurse & $7(70)$ & $9(90)$ & .29 \\
$N$ Respondents (\%) & $10(83.3)$ & $10(100)$ & \\
General Practitioner & $6(75)$ & $9(90)$ & .41 \\
$N$ Respondents (\%) & $8(66.6)$ & $10(100)$ & \\
Paediatrician & &
\end{tabular}

TABle 8: Vignettes.

\begin{tabular}{lccc}
\hline & \multicolumn{3}{c}{ Partially/fully correct } \\
Vignette & $\begin{array}{c}\text { Vietnamese } N(\%) \\
N \text { Respondents } \\
(\%)=11(91.6)\end{array}$ & $\begin{array}{c}\text { Australian } N(\%) \\
(\%)=10(100)\end{array}$ & $P$ \\
\hline Diarrhoea & $10(90.9)$ & $10(100)$ & .52 \\
Picky eater & $5(45.5)$ & $9(90)$ & .043 \\
Excessive milk & $9(81.8)$ & $10(100)$ & .26 \\
Feeding & $7(63.6)$ & $10(100)$ & .06 \\
Delayed walker & $3(27.3)$ & $5(50)$ & .27 \\
Delayed language & $4(40)$ & $6(60)$ & .21 \\
Tummy time & $7(58.3)$ & $8(80)$ & .27 \\
Reading & $6(54.5)$ & $10(100)$ & .023 \\
Cleanliness & $11(100)$ & $10(100)$ & .50 \\
Tantrum & $10(90.9)$ & $10(100)$ & .52 \\
\hline
\end{tabular}

study: utilisation of child health services and perceptions of parenting roles, child health, and child development.

4.1. Utilisation of Health Services. The majority of the Vietnamese study participants had Vietnamese family doctors, with whom they primarily consulted for issues relating to their children's health. Very few were familiar with the services provided by the Community Health Centre, apart from the BCG Immunisation Clinic, antenatal clinic, and the Home Visiting Program. In Queensland infants with parents from an Asian background are offered BCG vaccine to protect them from contracting tuberculosis, and information about where to obtain immunisations is given to parents at the time of discharge from the maternity hospital. The Home
Visiting Program at the time of this study was offered to all first-time mothers who requested the service or who were referred from the maternity hospital after giving birth.

The Vietnamese focus group participants expressed a wish for more home visiting services, to obtain assistance and advice about their infants' feeding and sleeping. However, Vietnamese questionnaire participants were less familiar with the role health professionals such as therapists, social workers, and community nurses. This is not surprising, considering that these professional roles do not exist in the traditional Vietnamese health system. Vietnamese participants first consulted their relatives and then their Vietnamese family doctor, for child health-related problems. Many preferred to delay and see their general practitioner rather than attend a community health centre and utilise the services of an interpreter. The vast majority of questionnaire participants in both groups gave the response "never needed their service" as a reason for never using a particular health service. The rest gave reasons of "do not know about them" and "too expensive", but nobody gave reasons of "transport problems", "language", "culture", or any other reason. Of those who had utilised other health services (therapy services, paediatricians, dentists and optometrists) the Vietnamese participants were less satisfied than Australian participants.

Several Australian studies have explored health concerns and barriers to accessing health services in Vietnamese families and arrived at similar conclusions as our study [1012]. These studies concluded that Vietnamese participants were highly concerned about their children's general health, growth, and development and nutrition [11].

Similar to the findings from this study, some studies found that Vietnamese-born immigrants expected from community health centres such services as home visiting, advice and information, health checks, and bilingual professionals [11, 12], but only $30 \%$ of Vietnamese people were aware of, and $10 \%$ utilised community health services [10]. Reasons given by participants in these studies for not utilising health services again were language barriers, inappropriate manner, lack of cultural sensitivity, transportation barriers, and waiting time $[10,11]$. Health professionals interviewed perceived that barriers to Vietnamese people accessing services included culture, language, transport, privacy, and lack of understanding of preventative health $[10,11]$. Maltby's study found Vietnamese parents to be less comfortable with services where the use of interpreters was required [10]. High quality of "care" was perceived as being kept informed of what was going on [10]. Another barrier to accessing health services may be traditional health beliefs.

Vietnamese people tend to define their health problems in terms of physical symptoms, including emotional or psychological disturbances, which avoids the stigmata of mental illness [26]. Initially symptoms are self-managed by adopting Chinese medical tenets and practices: for example, dermal practices such as Cao Gioa and adjustment of diet between "hot" and "cold" foods to balance humoral forces of yin and yang $[7,26]$. When self-care does not work, they seek a medical doctor to get medicine for the symptoms. If they go to a doctor and do not get medicine, they often feel 
TABLe 9: Causes of conditions.

\begin{tabular}{|c|c|c|c|c|}
\hline Condition & Cause & Vietnamese $N(\%)$ & Australian N (\%) & $P$ \\
\hline \multirow{4}{*}{ ADHD } & Upbringing & $4(44)$ & 0 & .029 \\
\hline & Preservatives & $3(27.3)$ & $6(60)$ & .24 \\
\hline & Previous life & 0 & 0 & - \\
\hline & Genetics & $3(27.3)$ & $8(80)$ & .043 \\
\hline$N$ respondents $(\%)$ & & $8(66.7)$ & $9(90)$ & \\
\hline \multirow{4}{*}{ Asthma } & Genetics & $7(63.6)$ & $10(100)$ & .055 \\
\hline & Catch it & $1(9.1)$ & 0 & .52 \\
\hline & Preservatives & 0 & $2(20)$ & .21 \\
\hline & Cold weather & $7(64)$ & $4(36)$ & .14 \\
\hline$N$ respondents $(\%)$ & & $11(91.7)$ & $10(100)$ & \\
\hline \multirow{4}{*}{$\mathrm{CP}$} & Genetics & $1(9.1)$ & $8(80)$ & .004 \\
\hline & Diet & $3(27.3)$ & $3(30)$ & .19 \\
\hline & Birth trauma & $5(62.5)$ & $3(30)$ & .18 \\
\hline & Previous life & $1(13)$ & 0 & .44 \\
\hline$N$ respondents $(\%)$ & & $8(66.7)$ & $10(100)$ & \\
\hline \multirow{4}{*}{ Epilepsy } & Previous life & $1(11.1)$ & 0 & .47 \\
\hline & Abortion & 0 & 0 & - \\
\hline & Head injury & $5(56)$ & $6(55)$ & .59 \\
\hline & Genetics & $5(56)$ & $9(82)$ & .26 \\
\hline$N$ respondents $(\%)$ & & $9(75)$ & $10(100)$ & \\
\hline \multirow{4}{*}{ Mental illness } & Previous life & 0 & 0 & .21 \\
\hline & Genetics & $3(25)$ & $9(90)$ & .017 \\
\hline & $\begin{array}{l}\text { Psychological } \\
\text { trauma }\end{array}$ & $6(66.7)$ & $8(80)$ & .44 \\
\hline & Abortion & $2(22.2)$ & 0 & .21 \\
\hline$N$ respondents (\%) & & $9(75)$ & $10(100)$ & \\
\hline
\end{tabular}

TABLE 10: Summary of main study findings.

Vietnamese participants compared with Australian participants

(1) Have different perceptions of early childhood development.

(2) Stigmatise mental and physical conditions more.

(3) Regard general practitioner as the primary as first point of call for children's health issues.

(4) Have little knowledge of available community child health services and do not tend to utilise these services apart from home visiting and antenatal services.

(5) Have little knowledge of allied health professionals' roles.

(6) Are less satisfied with community health services they have had contact with previously.

TABLE 11: Implications of study findings.

(1) Structures questionnaire study methodology may not be appropriate for study type.

(2) More qualitative methodology such as focus group discussions or unstructured interviews may be preferable.

(3) Potential differences in perceptions of the Vietnamese and Australian parents regarding child development warrant further study.

(4) Reasons for Vietnamese parents not accessing community child health services warrant further study.

"cheated" [26]. Western medicines are used more often for infectious diseases, and traditional medicine is preferred for digestive problems, insomnia, and colds [27].

A study into Vietnamese-born parents' oral health practices [13] concluded that Vietnamese parents in Australia retain traditional oral health practices and utilised oral health and dental services only when symptoms arose (e.g., toothache), rather than in a preventative manner. In addition to the lack of awareness of preventative dental health, the study parents cited language difficulties as being a major 
barrier to accessing school-based oral health programs for their children [13].

Australian research into use of children's hospital services by families from culturally and linguistically diverse (CALD) backgrounds found that CALD families were very satisfied with their children's care, though they also tended to not complain or express dissatisfaction for fear of repercussion or appearing to stand out [28]. The major barrier to accessing services was not just language but communication issues, which resulted in cultural mismatches. Issues from a patient or parent perspective included ability to express oneself clearly, understanding medical jargon, and communicating effectively with health professions [28]. Staff faced barriers in seeking to understand family needs and impart health information [28]. Interpreter services in part ameliorated these communication issues but the study raised concerns about appropriate use of and access to interpreter services [28].

4.2. Perception of Parenting Roles, Child Development, Child Health, Illness, and Disability. Both Vietnamese and Australian questionnaire participants recognised when to seek medical attention for an acute illness and both groups understood principles of infant nutrition.

Whilst there was no difference between the age (around six months) at which participants in the two focus groups introduced solid foods, the Vietnamese focus group participants tended to delay independent feeding, preferring to wait until the child was old enough to "not make a mess" (around 2 years). This was also consistent with the result from the feeding vignette in the questionnaire, which only $63.6 \%$ of Vietnamese study participants answered correctly. The focus group discussions found that the Vietnamese participants tended to allow their children to play with any toys that they wanted, whereas the Australian participants bought what they perceived to be educational toys. This was also consistent with the result of the reading vignette in which there was a difference between the two groups in correct responses, with only $54.5 \%$ of the Vietnamese group responding that they would read more to their child, and the rest responding that they would buy more books but let the child play with what they wanted.

The Vietnamese questionnaire participants were less likely to encourage "tummy time" in play than the Australian participants. Tummy time refers to placing the infant in the prone position during play and is thought to be important in encouraging development of crucial weight bearing upper body and upper limb muscles and consequently gross motor skills, such as pushing up and crawling [29]. Wise and Da Silva in their report for the Australian Institute of Family Studies (AIFS) described parenting as encompassing a number of different aspects, including beliefs, values, goals, and behaviours [30]. Each of these dimensions can be influenced by child characteristics, parent characteristics, physical and social context, and child rearing customs [30]. Harkness and Super [31] coined the term "parental ethnotheories" to help explain cultural differences in parenting. Ethnotheories are collective beliefs held by a cultural group about children's development and behaviour. Aspects of childrearing (e.g., Parenting goals, discipline practices, and beliefs about child development) are influenced by the characteristics of "individualistic" (Westernised) and "collectivist" (traditional) societies [32]. Individualistic societies value autonomy and independence and encourage children to demonstrate initiative from an early age, as well as honest expression of emotion [30]. In collectivist societies, children are taught to place community responsibility above individual interest, and emotional control is emphasised [30]. Parents of Anglo-Celtic origin often hold earlier expectations for development than parents from other cultures [30]. Acculturation as in the case of immigration can moderate the influence of culture on childrearing [30]. The findings from this study support the notion that Vietnamese parents tend to expect their children to achieve independent developmental milestones such as gross motor skills, and feeding skills, at a later age than Anglo-Australian parents, and that child rearing practices and beliefs of Vietnamese parents are influenced by collectivist philosophy.

Research has examined differences in the way various cultures parent and how this may impact on the child's development [33-36]. This literature indicates that most infants achieve major developmental milestones at similar ages [33], but developmental rate and more subtle aspects of development may differ between different cultures and ethnic groups $[34,36]$. Coll [35] in a review of this literature concluded that differences in parental goals, perceptions, and interactions with infants and children result in alternative or different pathways, which are within a normal range of development.

Research undertaken by Wise and colleagues for the AIFS has studied cultural variations in parenting beliefs and behaviours between Anglo-Australian parents, Vietnamese parents, and Somali parents [30]. Main findings of relevance were that Anglo parents valued independence in the child significantly more than Vietnamese parents, and child compliance was valued significantly more by Vietnamese parents than Anglo parents [30].

Vietnamese-focus group participants tended to use "softer" methods of explaining rather than "time-out". This is consistent with literature on more permissive and tolerant parenting of younger children in the Vietnamese culture [18]. The Australian focus group participants primarily had infants; so this topic was not as relevant to them. In contrast, the majority of questionnaire participants in both groups responded that they would use a "softer method" of explanation rather than "time-out" for the vignette about the child having a tantrum.

Vietnamese focus group and questionnaire participants were more likely to attribute nonmedical rather than medical factors as being causative for medical and neurodevelopmental conditions such as ADHD, epilepsy, and CP. One Vietnamese questionnaire respondent perceived "what you did in a previous life" to be a cause of epilepsy and CP, and another person perceived that attempted abortion was a cause of mental illness in a mother. One Vietnamese focus group participant perceived that Karma, attempted abortion, and taking the oral contraceptive pill were also causes of 
mental illness in children. These findings are consistent with the current literature, which indicates that Asians living in Western countries tend to stigmatise disability and mental illness more than their Western counterparts [3743]. One such study explores the perceptions of various groups of ethnic university students and found that AsianAmericans, particularly those Asian born, saw physical and mental disabilities as being more stigmatised than did African-Americans, Latin-Americans, and EuropeanAmericans [38]. An Australian study found that Asian students, especially those who spoke a language other than English at home, were more negative toward and less knowledgeable about mental illness than EuropeanAustralian students [39].

Another Australian study, which explored parental perceptions of child psychopathology and disease causation, of Vietnamese parents living in Perth, found that the majority of parents $(75.5 \%$ to $78.1 \%)$ thought that child mental illness was caused by biological/chemical imbalance in the brain, trauma or bad experiences, and a metaphysical or spiritual imbalance. $57.2 \%$ endorsed a genetic or hereditary cause, and $17.6 \%$ indicated karma as the cause of child mental illness.

One reason for Vietnamese immigrants' more negative perception of mental illness may be the Confucian tradition of sacrificing individual needs to that of the overall group needs [40]. Since a person with a disability may not contribute to the collective good in a way that a healthy person might contribute [40], they are perceived as less important. A strong emphasis on conformity and a general intolerance of differences in Asian cultures may also be a relevant factor $[41,42]$. Another contributing factor may be the commonly held belief that mental illness is a manifestation of weakness of the mind [37]. A psychiatrist is referred to as either a "nerve doctor" or a "doctor for the insane", which emphasises Vietnamese people's beliefs that mental illness are "bizarre and out of this world" in nature [37]. As a result of these beliefs, Vietnamese people rarely seek out mental health services [37].

4.3. Conflicting Study Findings. There are a number of possible explanations for conflicting findings from the Vietnamese focus group discussions and questionnaire. Firstly, there may be inherent differences in these two groups, which influence perceptions regarding these issues. Participants from both groups were mostly recruited from the Centre's BCG Immunisation Clinic. However sociodemographic information about the focus group participants would have been helpful in comparing these two groups. The second explanation is measurement or information bias due to two different methodological approaches, thus eliciting different responses. Of note the questionnaire response rate in the Vietnamese group was only $7 / 12$ compared with $11 / 11$ for the Australian group. The literature on cross-cultural research supports this explanation by indicating that focus groups or face-to-face interviews may be a more effective data collection method than structured questionnaire, as they can foster trust and allow participants to describe their view and experiences in a context in which cultural nuances can be recognised [25]. The Parenting-21 Study conducted by the AIFS utilised a semistructured questionnaire which was completed by the interviewer in a face-to-face interview [16]. The third possibility is that the sample size is too small for findings from the focus groups and questionnaires to be valid.

4.4. Study Limitations and Generalisability. There are limitations to this study, which may contribute to conflicting study findings. The main source of potential bias was differences in recruitment method for Vietnamese and Australian participants. The Vietnamese participants attended the BCG Immunisation clinic, whereas the Australian participants were attendants of the parents' groups and baby clinics. This problem with recruitment was unavoidable, as no immunisation clinic exists for Caucasian Australian children at the Centre, and no existing Vietnamese clients utilised other services at the Centre.

Utilisation of an inappropriate data collection method, of a structured questionnaire, may have resulted in measurement bias as it does not give the flexibility to express views and perceptions fully and can misinterpret cultural nuances [25].

The majority of questionnaire participants both Vietnamese and Anglo-Australian were from a low sociodemographic background with no tertiary education and low income. The demographic profile of study participants is representative of Inala's population [44]. For example, in the 2001 Census, the median weekly family income for Inala was from $\$ 500$ to $\$ 599$, whereas that for Australia is from $\$ 800$ to $\$ 999$.

However these findings cannot be generalizable to AngloAustralian or Vietnamese parents from higher sociodemographic backgrounds. In addition although 50\% of the Vietnamese questionnaire participants had been in Australia for eight years or more, most of them rated their spoken English ability and understanding and ability to read English as "all right" or "very little or not at all". Thus the study results are not necessarily generalizable to Vietnamese immigrants who are more acculturated into Australian society.

\section{Conclusions}

Findings have highlighted issues relating to Vietnamese parents' perceptions and beliefs regarding children's health, illness, and development, as well as child health service utilisation, which have not yet been extensively studied in the past. Vietnamese parents in comparison to Anglo-Australian parents have a more collectivist view of child rearing and place less expectation on infants and young children to achieve independence. Vietnamese parents tend to attribute nonbiological or metaphysical causation such as Karma to mental illness or neurodevelopmental conditions such as epilepsy, ADHD, and CP. They have less familiarity with the range of health services available for their children and are generally less satisfied with the English-speaking mainstream services that they have utilised in the past.

There is a need for further examination of these issues with future studies, using more culturally appropriate 
qualitative methods and larger sample sizes. A better understanding of these issues within Vietnamese Communities in Brisbane and throughout Australia will result in the delivery of more culturally sensitive and effective health services to children and families within these communities.

\section{Acknowledgments}

The author would like to acknowledge the supervision and support of Dr. Alison Harris, the assistance of Dr. Linda Shields in the initial development phase, and the assistance of Dr. Michael O'Callaghan. Her thanks go to Dr. Anne George and Dr. Cherylynn Bassani for reviewing manuscript drafts. She would like to thank Virginia Leyden for her assistance in the focus group discussions, recruitment, and questionnaire completion. Last but not least, she would like to thank the staff at Inala Child Youth and Family Health for their enthusiasm, dedication, and support. This study was supported by Queensland Health, Queen Elizabeth II Hospital Health District (QEII). Ethics approval was obtained through the Princess Alexandra Hospital Ethics Committee.

\section{References}

[1] C. Chu, "Cross-cultural health issues in contemporary Australia," Ethnicity \& Health, vol. 3, no. 1-2, pp. 125-134, 1998.

[2] K. Newbold and J. Danforth, "Health status and Canada's immigrant population," Social Science \& Medicine, vol. 57, no. 10, pp. 1981-1995, 2003.

[3] M. Beiser, F. Hou, I. Hyman, and M. Tousignant, "Poverty, family process, and the mental health of immigrant children in Canada," American Journal of Public Health, vol. 92, no. 2, pp. 220-227, 2002.

[4] G. Flores, H. Bauchner, A. R. Feinstein, and U. S. Nguyen, "The impact of ethnicity, family income, and parental education on children's health and use of health services," American Journal of Public Health, vol. 89, no. 7, pp. 1066-1071, 1999.

[5] R. M. Weinick, S. H. Zuvekas, and J. W. Cohen, "Racial and ethnic differences in access to and use of health care services, 1977 to 1996," Medical Care Research and Review, vol. 57, supplement 1, pp. 36-54, 2000.

[6] S. G. Brink and P. R. Nader, "Patterns of primary care utilization in a triethnic urban population of school children," Medical Care, vol. 19, no. 6, pp. 591-599, 1981.

[7] C. N. H. Jenkins, T. Le, S. J. McPhee, S. Stewart, and N. T. Ha, "Health care access and preventive care among Vietnamese immigrants: do traditional beliefs and practices pose barriers?" Social Science \& Medicine, vol. 43, no. 7, pp. 1049-1056, 1996.

[8] G. A. Gellert, R. M. Maxwell, K. V. Higgins, K. K. Mai, and R. Lowery, "Barriers to health care access and utilization among vietnamese Americans in Southern California," Health \& Place, vol. 1, no. 2, pp. 91-99, 1995.

[9] S. M. Yu, Z. J. Huang, and G. K. Singh, "Health status and health services access and utilization among Chinese, Filipino, Japanese, Korean, South Asian, and Vietnamese children in California," American Journal of Public Health, vol. 100, no. 5, pp. 823-830, 2010.

[10] H. Maltby, "Health promotion for Vietnamese women and their families," Nursing Standards, vol. 12, no. 32, pp. 40-43, 1998.
[11] R. Islip, "Consultation with Vietnamese/ Chinese families about health concerns and health services," in Proceedings of the Health, Culture and Well-Being Asian-Pacific Paediatric Nursing Conference, pp. 30-40, Melbourne, Australia, June 1995.

[12] H. Phung and M. Tran, Vietnamese community health needs assessment, M.S. thesis, University of Queensland, 1994.

[13] C. F. Lamb and C. Phelan, "Cultural observations on Vietnamese children's oral health practices and use of the child oral health services in central sydney: a qualitative study," Australian Journal of Primary Health, vol. 14, no. 1, pp. 75-81, 2008.

[14] M. Cheung and S. Nguyen, "Parent-child relationships in Vietnamese-American families," in Culturally Diverse ParentChild and Family Relationships: A Guide for Social Workers and Other Practitioners, N. B. Webb, Ed., pp. 261-281, Columbia University Press, New York, NY, USA, 2001.

[15] J. C. Rossiter, "Promoting breast feeding: the perceptions of Vietnamese mothers in Sydney, Australia," Journal of Advanced Nursing, vol. 28, no. 3, pp. 598-605, 1998.

[16] V. Kolar and G. Soriano, Parenting in Australian Families: A Comparative Study of Anglo, Torres Strait Islander, and Vietnamese communities, Australian Institute of Family Studies, Melbourne, Australia, 2000.

[17] P. Liamputtong-Rice, K. Cape, N. Doan et al., Asian Mothers, Western Birth. Pregnancy, Childbirth and childrearing: The Asian Experience in an English-speaking Country, Ausmed Publications, Melbourne, Australia, 2nd edition, 1994.

[18] V. Nguyen and M. Ho, "Vietnamese-Australian families," in Families and Cultural Diversity in Australia, R. Hartley, Ed., pp. 216-241, Allen \& Unwin, 1995.

[19] F. Papps, M. Walker, A. Trimboli, and C. Trimboli, "Parental discipline in Anglo, Greek, Lebanese, and Vietnamese cultures," Journal of Cross-Cultural Psychology, vol. 26, no. 1, pp. 49-64, 1995.

[20] R. Ranford, "Implications and considerations of family rearing practices," Professional Digest, vol. 3, no. 2, pp. 31-33, 1992.

[21] Australian Bureau of Statistics, "Australia social trends 2001.Population-population composition: Asian-born Australians," Tech. Rep. 1269.0, 2002.

[22] D. Polit, C. Beck, and B. Hungler, Essentials of Nursing Research: Methods, Appraisal and Utilisation, Lippincott, Philadelphia, Pa, USA, 5th edition, 2001.

[23] R. Hughes and M. Huby, "The application of vignettes in social and nursing research," Journal of Advanced Nursing, vol. 37, no. 4, pp. 382-386, 2002.

[24] A. Chang, J. Chau, and E. Holroyd, "Translation of questionnaires and issues of equivalence," Journal of Advanced Nursing, vol. 29, no. 2, pp. 316-322, 1999.

[25] A. Neufeld, M. J. Harrison, K. D. Hughes, D. Spitzer, and M. J. Stewart, "Participation of immigrant women family caregivers in qualitative research," Western Journal of Nursing Research, vol. 23, no. 6, pp. 575-591, 2001.

[26] M. A. Muecke, "Caring for Southeast Asian refugee patients in the USA," American Journal of Public Health, vol. 73, no. 4, pp. 431-438, 1983.

[27] P. H. Stephenson, "Vietnamese refugees in Victoria, B.C.: an overview of immigrant and refugee health care in a mediumsized Canadian urban centre," Social Science \& Medicine, vol. 40, no. 12, pp. 1631-1642, 1995.

[28] S. Chalmers and A. Rosso-Buckton, "Are you talking to me? Negotiating the challenge of cultural diversity in children's 
health care,” Tech. Rep., Centre for Cultural Research, University of Western Sydney, 2008.

[29] L. Bly, Motor Skills Acquisition in the First Year. An Illustrated Guide to Normal Development, Therapy Skill Builders, Tuscan, Ariz, USA, 2000.

[30] S. Wise and L. Da Silva, Australian Institute of Family Studies. Differential Parenting of Children from Diverse Cultural Backgrounds Attending Child Care, Australian Institute of Family Studies, Melbourne, Australia, 2007.

[31] S. Harkness and C. M. Super, "Parental ethnotheories in action," in Parental Belief Systems: The Psychological Consequences for Children, I. E. Sigel, A. V. McGillicuddy-De Lisi, and J. J. Goodnow, Eds., pp. 373-392, Lawrence Erlbaum Associates, Hillsdale, NJ, USA, 1992.

[32] R. L. Harwood, A. Schoelmerich, P. A. Schulze, and Z. Gonzalez, "Cultural differences in maternal beliefs and behaviors: a study of middle-class Anglo and Puerto Rican mother-infant pairs in four everyday situations," Child Development, vol. 70, no. 4, pp. 1005-1016, 1999.

[33] J. Kagan, "Canalization of early psychological development," Pediatrics, vol. 70, no. 3, pp. 474-483, 1982.

[34] E. Werner, Cross-Cultural Child Development, Brooks/Cole, San Francisco, Calif, USA, 2006.

[35] C. T. Garcia Coll, "Developmental outcome of minority infants: a process-oriented look into our beginnings," Child development, vol. 61, no. 2, pp. 270-289, 1990.

[36] P. Leiderman, S. Tulkin, and A. Rosenfeld, Culture and Infancy: Variation in the Human Experience, New York Academic Press, New York, NY, USA, 1977.

[37] D. Nguyen, "Culture shock- a review of Vietnamese culture and its concepts of health and disease," Western Journal of Medicine, vol. 142, no. 3, pp. 409-412, 1985.

[38] C. L. Saetermoe, D. Scattone, and K. H. Kim, "Ethnicity and the stigma of disabilities," Psychology \& Health, vol. 16, no. 6, pp. 699-713, 2001.

[39] C. Fan, "A comparison of attitudes towards mental illness and knowledge of mental health services between Australian and Asian students," Community Mental Health Journal, vol. 35, no. 1, pp. 47-56, 1999.

[40] F. Chan, J. Hedl, H. Parker, C. Lam, T. Chan, and B. Yu, "Differential attitudes of Chinese students toward people with disabilities: a cross-cultural perspective," International Journal Social Pysychiatry, vol. 34, pp. 206-217, 1988.

[41] M. Westbrook, V. Legge, and M. Pennay, "Attitudes towards disabilities in a multicultural society," Social Science \& Medicine, vol. 36, no. 5, pp. 615-623, 1993.

[42] Y. Kojima, "Disabled individuals in Japanese society," Rehabilitation World, vol. 3, no. 2, pp. 18-25, 1977.

[43] R. S. McKelvey, L. V. Baldassar, D. L. Sang, and L. Y. N. N. Roberts, "Vietnamese parental perceptions of child and adolescent mental illness," Journal of the American Academy of Child \& Adolescent Psychiatry, vol. 38, no. 10, pp. 1302-1309, 1999.

[44] D. Trewin, "Census of population and housing: selected social and housing characteristics, Australia 2001," Tech. Rep. 2015.0, Australian Bureau of Statistics, Canberra, Australia, 2003. 


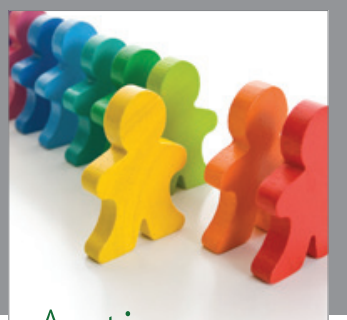

Autism

Research and Treatment
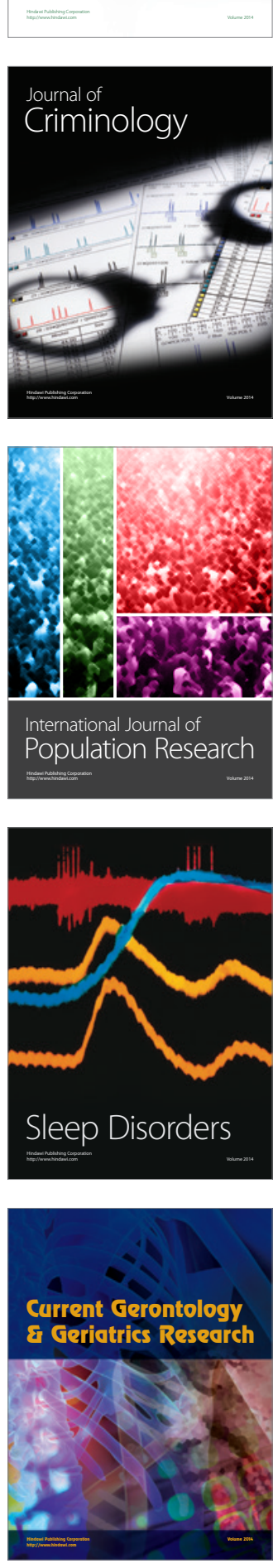
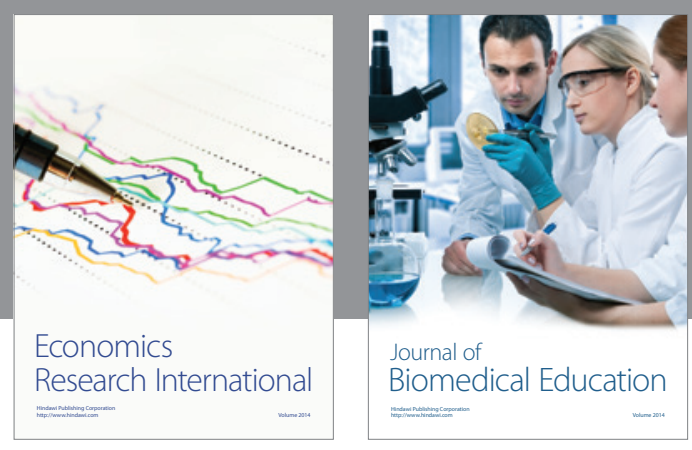

Journal of

Biomedical Education

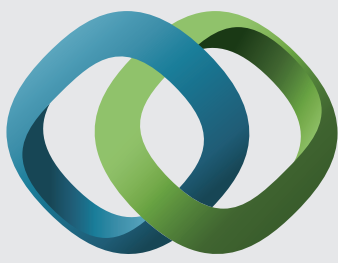

\section{Hindawi}

Submit your manuscripts at

http://www.hindawi.com
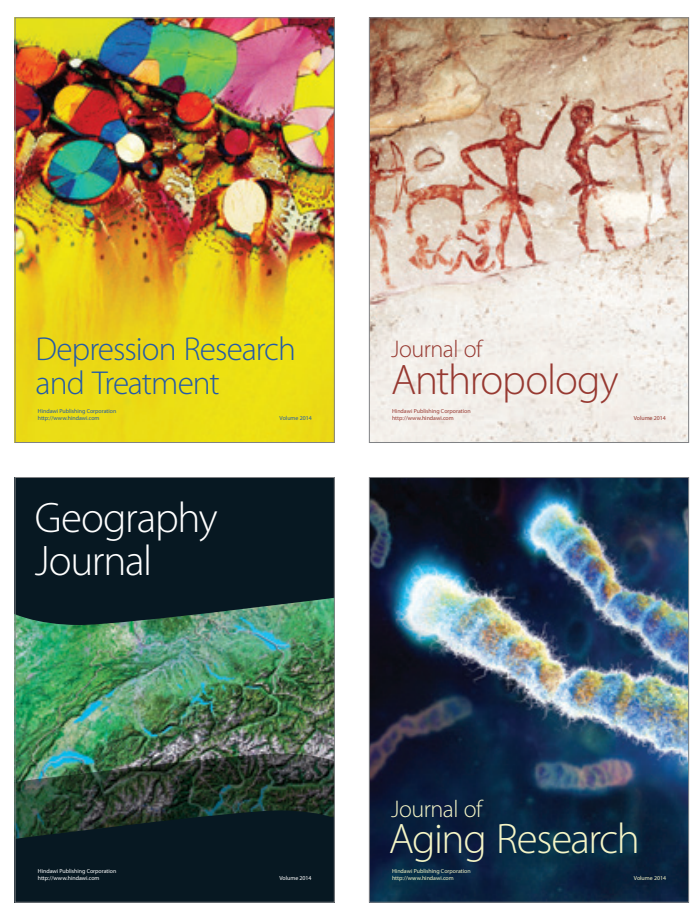

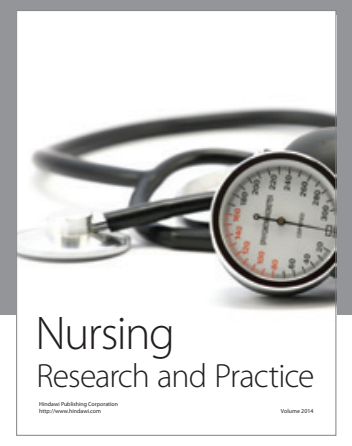

Nursing

Research and Practice

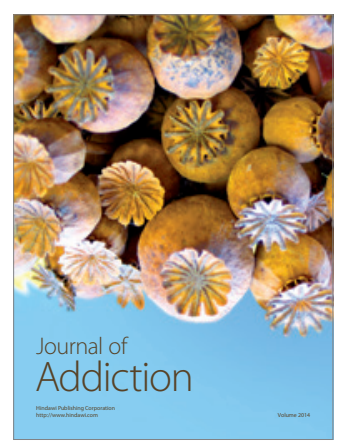

Child Development

Research

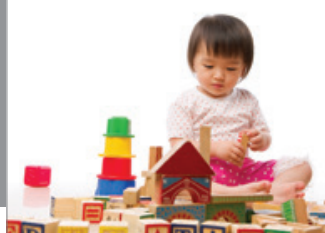

迥
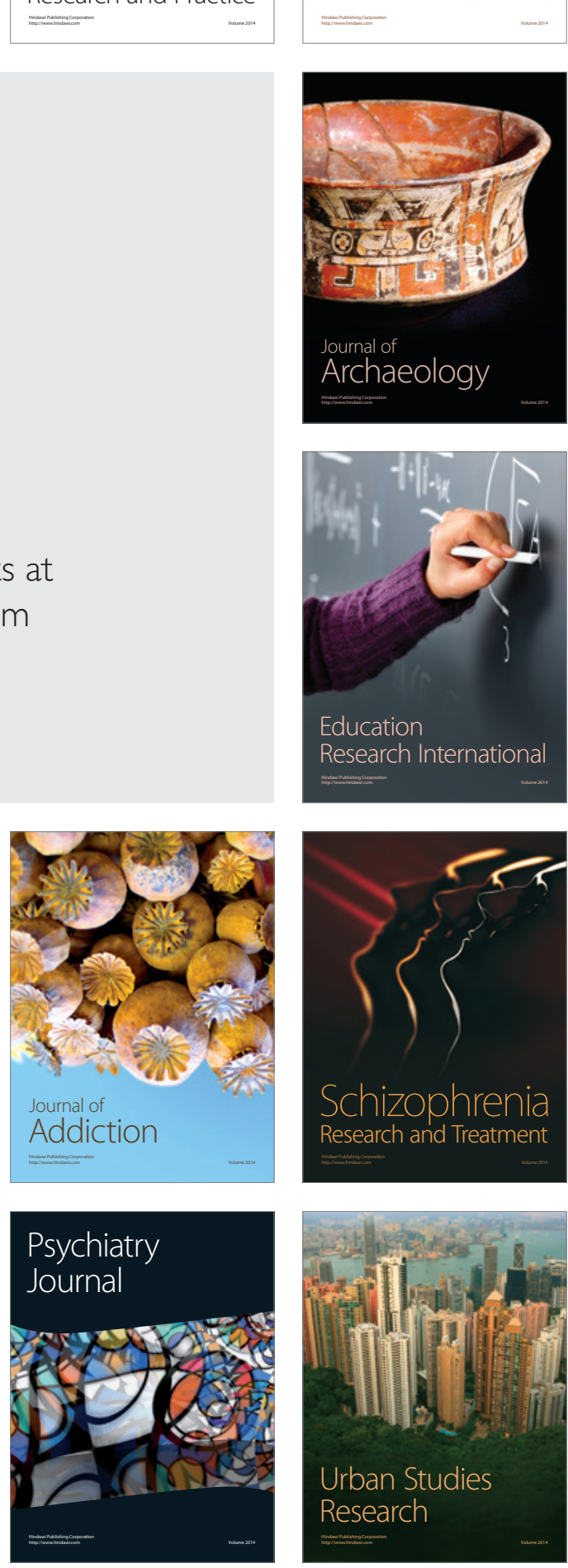